\title{
Retention of fear'
}

Retention of fear decreased over intervals of $22 \mathrm{hrs} ., 30$, 60 and 90 days after classical fear conditioning. Fear retained at 22-hr. and 30-day intervals was sufficient to provide the basis for new instrumental learning.

Campbell \& Campbell (1962) reported decreasing retention of fear by rats classically conditioned during infancy in one side of a two-compartment cage. Retention of fear was measured by the amount of time, in a $60-$ min. period, that $S$ avoided the previouslyshocked compartment by staying in the compartment where shock had never occurred. This technique provided no information as to whether or not the amount of fear retained was sufficient to motivate and reinforce new instrumental learning. The present study provides such information. Briefly, fear-conditfoning procedures were followed, after delay intervals, by training without shock in which a hurdle-jumping response permitted $S$ to escape the presumably feareliciting cues. The acquisition of the hurdle-jumping response was the index of whether or not sufficient fear was retained to provide for new, instrumental learning.

Further, this study investigated effects of stimulus complexity and stimulus generalization on retention of fear. McAllister \& McAllister $(1962,1963)$ demonstrated the influence of these stimulus variables on the acquisition of a hurdle-jumping response, motivated and reinforced by fear. There is no reported information on the effects of stimulus complexity and stimulus generalization on the retention of fear.

\section{Method}

Apparatus. The apparatus has been described in detail by McAllister \& McAllister (1963). Briefly, a separate conditioning box and a hurdle-jumping (HJ) apparatus were used. The HJ apparatus consisted of a white start box separated from a gray safe box by a guillotine door and a hurdle. The start-box floor consisted of brass rods which could be electrified successively with $90 \mathrm{~V}$, at the rate of 2 impulses per rod per sec., by a commutator driven by a constantspeed motor. The circuit resistance was 100,000 ohms in series with each $\mathrm{S}$. The separate conditioning box was constructed similarly and was wired in parallel with the start box. These boxes have been compared by McAllister \& McAllister (1963). The safe box of the HJ apparatus had a masonite floor which depressed under S's weight.

Each of these boxes had hinged lids which contained light sources for the intertrial illumination (7 ft.-c.). The lids of the start box and the separate conditioning compartment also contained light sources for the presentation of a discrete CS (an increase in illumination from $7 \mathrm{ft}$. $-\mathrm{c}$. to $115 \mathrm{ft}$.-c.).

Subject, design, and procedure. Ss were 128 experimentally naive, female, hooded rats. At 29-33 days of age, Ss were randomly assigned to experimental groups and were treated in pairs throughout the experiment.

Two days of handling and exploration of the HJ apparatus were followed by a day of 35 fear-conditioning trials and, then, two consecutive days of 25 hurdle-jumping trials per day.

On the conditioning day, a pair of Ss received 35 conditioning trials according to either a forward conditioning (FC) or a backward conditioning (BC) paradigm, one $S$ in the start box of the HJ apparatus, the other $S$ in the separate conditioning box. An FC trial consisted of a 6-sec. presentation of the discrete CS (light) overlapped, in the final 2 sec., by a $90-\mathrm{V}$ UCS; a BC trial consisted of 2 sec. of UCS followed, 15 sec. later, by 6 sec. of the discrete CS (light).

Since the static apparatus cues were always present during conditioning, FC procedures presumably conditioned fear to the complex of discrete CS (light) and static cues; BC procedures presumably conditioned fear to the static cues alone. The intertrial interval during conditioning was $2 \mathrm{~min}$.

Hurdle-jumping training was initiated $22 \mathrm{hr}, 30$, 60 , or 90 days after the start of conditioning. A hurdlejumping trial began with the insertion of $S$ into the HJ-apparatus start box. Ten sec. later, the guillotine door was raised, activating the discrete CS (light) and a .01 sec. timer. If $\mathrm{S}$ jumped the hurdle, the safe box floor was depressed, terminating the CS (light) and timer, and $S$ was removed after 10 sec. If $S$ failed to jump within 60 sec., $S$ was removed from the start box, CS and timer remaining on, and a latency of $60 \mathrm{sec}$. was recorded.

The effects of stimulus complexity on retention of fear were evaluated by comparing FC and BC groups on hurdle-jumping performance. The effects of stimulus generalization were evaluated by comparing hurdle-jumping performance between groups fear-conditioned in the HJ-apparatus start box (Original-CS groups) and groups fear-conditioned in the separate conditioning box (Generalized-CS groups).

\section{Resulis}

In Fig. 1, Retention Interval is the parameter; all other design factors are equally represented in each curve. Performance tended to increase over trial blocks and was inversely related to retention interval. An overall trend analysis showed significant F-values for Retention Interval ( $F=17.95, d f=3 / 240, p<.001)$, 


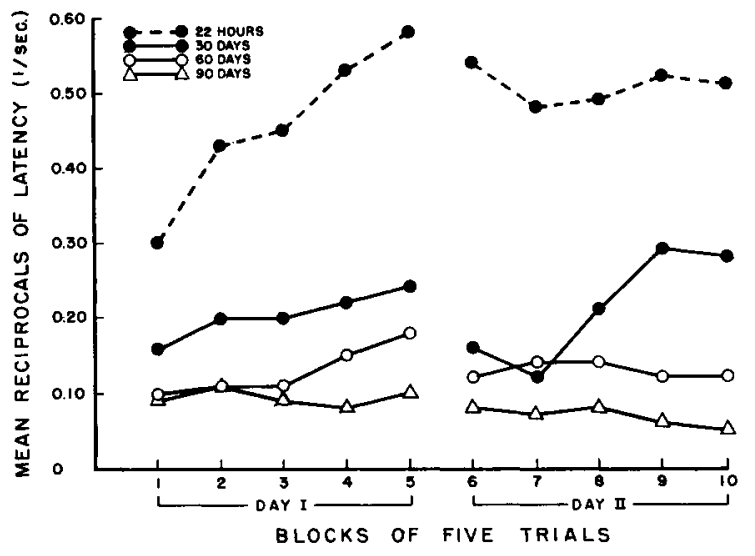

Fig. 1. Mean reciprocals of latency on Day 1 and on Day 2 of hurdle-jumping training for groups trained $22 \mathrm{hr} ., 30,60$, or 90 days after conditioning. The factors for Type of Conditioning and for Stimulus Generalization are equally represented in each curve.

Trial Blocks $(F=3.00, \mathrm{df}=9 / 880, p<.005)$, and Trial Blocks by Retention Interval $(F=2.00, d f=27 / 880, p<$ .01). Evaluation of the interaction showed that hurdlefumping performance increased reliably over trial blocks for the 22-hr. and 30-day groups (F=2.50, 2.00, df $=9 / 252, p=.01,<.05)$, but not for the 60 - or 90-day groups (Fs $<1$ ).

The F-values for the factors, Type of Conditioning (FC vs. BC) and for Stimulus Generalization (Original vs. Generalized CS) were non-significant. However, throughout training and at each retention interval, hurdle-jumping performance was superior for FC procecures over BC procedures and for the generalized CS condition over the original CS condition. Discussion

This study showed that fear retained $22 \mathrm{hr}$. and 30 days after conditioning was sufficient to provide the basis for new instrumental learning. Consistent with Campbell and Campbell's results, retention decreased with time.

Since Ss were fear-conditioned at the same age and were tested for retention after different intervals, the Ss' ages at the time of testing varied directly with the lengths of the retention intervals. In evaluating the possible effects of age-correlated factors (e.g., activity level), it was assumed that these factors would tend to facilitate acquisition performance of youngest Ss (the 22-hr. groups) more than that of the older Ss (the 30-, 60-, or 90-day groups) at the time of testing. Thus, 16 additional Ss received the same procedures as did the fear-conditioned (FC) Ss in the 22-hr. interval except that no shock was presented during "conditioning." The hurdle-jumping performance of the no-shock 22-hr. group showed no reliable increase over trials and was reliably inferior to that of the fear-conditioned 22-hr. group. Thus, at the 22-hr. interval, age factors contributed reliably less to hurdle-jumping performance than did fear conditioning.

On the plausible assumption that the hurdle-jumping performance of the non-shock 22-hr. group reflected the upper limit of performance determined solely by age factors, no non-shock Ss were treated at the $30-, 60-$, or 90-day intervals. Thus, at the 30-day interval the reliable increase in hurdle-jumping performance over trials probably occurred more on the basis of retention of fear than on the basis of agecorrelated mechanisms.

Finally, the failure to show reliable effects for stimulus complexity was inconsistent with the results of McAllister \& McAllister (1962) for adult rats. Since the procedures of this experiment were highly similar to those of McAllister \& McAllister (1962), this inconsistency may reflect differences between adult and infant rats in their conditionability to a discrete visual $\mathbf{C S}$.

\section{References}

Campbell, B. A., \& Campbell, E. H. Retention and extinction of learned fear in infant and adult rats. J. comp. physiol. Psychol., $1962,55,1-8$.

McAllister, W. R., \& McAllister, D. E. Role of CS and of apparatus cues in the measurement of acquired fear. Psychol. Rep., 1962, $11,749-756$.

McAllister, W. R., \& McAllister, D. E. Increase over time in the stimulus generalization of acquired fear. J. exp. Psychol., 1963, $65,576-582$.

\section{Notes}

1. Submitted in partial fulfillment of the M. A. degree from Syracuse University, 1964. The author is indebted to Drs. Wallace R. and Dorothy E. McAllister for their continual advice and support during this experiment. Grant No. MH-02064 from NIMH to the McAllisters provided partial funding for this study.

2. Now at Southern Methodist University. 